\title{
LA FRONTIÈRE FRANCO-ESPAGNOLE ET L'EUROPE : LE DÉPASSEMENT DES LIMITES ?
}

\section{INTRODUCTION}

La vogue des euro-régions, que sous-tend la force des mobilisations locales à travers toute l'Union européenne, semble révéler un rapport accru de ses ressortissants à la territorialité ; elle suggèrerait également une orientation politique du projet communautaire dans un sens différent de l'esprit initial des Traités de Rome, élaborés par des États et pour des États. En effet, le couple Europe/régions paraît maintenant indissociable : l'introduction du principe de subsidiarité et la création du Comité des Régions instillent dans les traités deux éléments fédéraux d'intégration à même de sortir les affaires locales du carcan État/collectivités territoriales, dans lequel elles sont encore conduites aujourd'hui. Il serait cependant prématuré, puisque l'aménagement du territoire reste du ressort des États, de parler d'une « Europe des régions », régions dont on peut en outre douter à l'heure actuelle de leurs capacités à devenir des acteurs à part entière de la «gouvernance européenne ${ }^{1}$. Il n'en reste pas moins que ces «eurorégions » qui fleurissent sur tout le Vieux Continent marquent une revanche de la géographie sur l'histoire, puisque les frontières, ces «produits de l'imaginaire » et de l'idéologie nationale, ne sont plus des limites intangibles, mais des zones de contact et de circulations ${ }^{2}$. De fait, ce n'est pas sur la frontière vue comme une ligne, mais au sein même de la région frontalière que les modalités d'intégration à l'Europe peuvent être le mieux observées. Cette approche, qui mêle histoire et anthropologie, rejoint les préoccupations des géographes qui s'intéressent au phénomène frontalier comme base de constructions régionales originales, en se fondant sur l'idée que la frontière déforme certaines interactions et produit des perceptions particulières de l'espace ${ }^{3}$.

Peut-on, pour autant, parler de nouvelles «solidarités de fait»? L'espace pyrénéen, particulièrement concerné par les programmes transfrontaliers lancés par la Commission européenne, dont le récent découpage «Sud-Ouest européen » du programme Interreg IIIb pour la période 2000-2006 est une illustration, est un bon cas d'étude pour vérifier la réalité de cette solidarité : fruit d'une politique récente, au regard d'autres mobilisations transnationales à l'œuvre dans l'Union européenne, cet espace frontalier met en scène des acteurs séparés depuis des siècles jusques et y compris, pour paraphraser Pascal, dans leur manière de raisonner ${ }^{4}$. Il s'agit dès lors de s'interroger sur cette « zone d'incertitude » qu'est la frontière, afin de voir si l'espace frontalier franco-espagnol va dans le sens de l'intégration, et si l'affirmation du « fait régional » facilite le rapprochement des peuples dans un lieu qui, par définition, marque une rupture : une «petite Europe » (concept qui désignerait une forme particulière d'attachement humain à un espace transnational) peut-elle naître entre France et Espagne, vérifiant la possibilité «d'inventer» un territoire par le biais de la solidarité régionale?

\footnotetext{
${ }^{1}$ Voir Patrick Le Galès, "Gouvernement et gouvernance des régions : faiblesses structurelles et nouvelles mobilisations », in Patrick Le Galès, Christian Lequesne, Les Paradoxes des régions en Europe, coll. Recherches, La Découverte, Paris, 1997, pp.237-263.

${ }^{2}$ Pour reprendre la terminologie des juristes, le concept de frontière «ligne de séparation » (Boundary) cède la place à celui de frontière «zone de contact» (Frontier), au sein de laquelle les États limitrophes ont des relations de contiguité qui donnent lieu à des avantages pour les nationaux respectifs qui y vivent. ${ }^{3}$ Voir à ce propos Michel Foucher, Fronts et Frontières, un tour du monde géopolitique, Fayard, Paris, 1991.

${ }^{4}$ «Plaisante justice qu'une rivière borne! Vérité au deçà des Pyrénées, erreur au delà », Pensées, Gallimard, Paris, p.294.
} 


\section{I- Europe et régions : un couple parfait ?}

\section{1) Les régions et le fédéralisme.}

Il faut, dans un premier temps, faire une digression sur le modèle fédéraliste, tel qu'il est défendu par les régions au niveau européen. En ce sens, leur discours s'oppose à celui des élites européennes, dans la manière de procéder, mais également à propos des buts à atteindre. Au plus haut sommet européen, depuis la création de la CECA (1951), les réflexions sur la CED (1950-1954) ou la mise en place de la CEE à la suite de la «relance européenne » (1955-1957), les thèses fonctionnalistes défendues par les Pères de l'Europe, et reprises par leurs héritiers «indignes », les technocrates ou « eurocrates », vont dans le sens d'une approche supranationale, conception purement juridique et institutionnelle, qui souhaite réaliser l'unité par le haut grâce à l' " effet de débordement » (spill-over effect ${ }^{5}$ ). Le mot lui-même mérite une petite explication, tant il est polysémique : popularisé par le fédéraliste Alexandre Marc lors du Congrès de La Haye en mai 1948, il sert à évoquer une réalité politique nouvelle, indépendante des structures nationales traditionnelles, placée au-dessus de celles-ci, permettant une pratique radicalement moderne de la politique à l'échelle du Vieux Continent. Le terme a cependant connu une postérité qui n'a pas rendu sa signification très claire : aussi bien employé par Robert Schuman au moment de la création de la CECA pour signifier que l'État continuait à maîtriser la politique européenne qu'il suscitait, que par le Général de Gaulle pour dénoncer la conspiration eurocratique qui menaçait la légitimité de chacun des États, ce concept fait aujourd'hui partie du vaste arsenal de termes politiques approximatifs qui servent à désigner un paysage politique européen qui luimême se cherche encore. Deux sens paraissent actuellement possibles et sont en concurrence :

-la supranationalité n'est pas forcément une négation de la souveraineté nationale, dans la mesure où elle peut résulter d'une délégation consentie par les États, à l'issue d'un processus de négociation de textes ratifiés par les parlements nationaux et ne porter que sur des secteurs limités : ainsi, le Traité CECA a mis en place une Haute Autorité, organe nommé d'un commun accord par les gouvernements et jouissant ensuite d'une totale indépendance à leur égard; cependant, ses pouvoirs de réglementation directe se limitent aux seuls secteurs du charbon et de l'acier: le principe de départ est que la souveraineté reste forcément absolue et indivisible.

-cela dit, la question supranationale ne se règle pas sur le court terme : modeste dans ses moyens, le projet européen est ambitieux dans ses objectifs ultimes (sécurité commune, développement mutuel) et peut masquer un processus de fédéralisation sur un temps indéfini (c'est pourquoi certains évoquent un « grignotage »).

\footnotetext{
5 Spill-Over Effect (Effet de Débordement): concept de base de la théorie néo-fonctionnaliste d'intégration européenne, énoncé en 1958 par l'Américain Ernst B. Haas. Une intégration décidée dans n'importe quel secteur, qu'il soit économique ou politique, entraîne des réactions dans les secteurs voisins de telle manière que l'effet induit est, à terme, une coopération renforcée. Cette intégration peut avoir une origine politique (CECA, Euro) ou peut être la conséquence d'une évolution contenue dans les Traités européens eux-mêmes (primauté du droit communautaire sur le droit national), qui elle-même entraîne la légitimation de nouveaux centres de décision (Comité des Régions, lobbying européen à Bruxelles). L'effet de débordement, parce qu'il ne peut être quantifiable, est peut-être à l'origine du «déficit démocratique » dénoncé depuis le Traité de Maastricht par ceux qui voudraient une intégration plus concertée et plus contrôlable politiquement par l'intermédiaire d'un Parlement européen renforcé (logique fédéraliste), au détriment d'une technocratie aux pouvoirs discrétionnaires.
} 
On a ici un résumé des deux approches par lesquelles la construction européenne est perçue du grand public : processus légitime, mais qui ne peut se passer des impulsions des États (modèle confédéral) ou bien processus mécanique, qui vise à inventer de toutes pièces une nouvelle forme de souveraineté par effet d'entraînement (modèle fédéral mais qui ne dit pas son nom).

Les régions tiennent un discours, en matière européenne, beaucoup plus particulariste, qui envisage certes l'unité européenne, mais fondée sur de fortes autonomies qui élargissent la base à mesure que le sommet s'élève plus haut : ce type de fédéralisme imagine une "communauté de communautés », prenant en compte le passé millénaire qui s'inscrit dans chaque paysage et chaque idiome selon la formule «l'unité dans la diversité ». Déjà, pour Jean Charles-Brun, «les nations fédérées [...] n'auront pas à renoncer à leur vie propre, à leur originalité. Rien de plus éloigné du magma européen, dont rêvent certains, que la pure conception proudhonienne. Elle va si loin là-dessus qu'elle veut, à l'intérieur de chaque État même, le maintien des nationalités qui la composent, et qu'elle se refuse à leur assimilation centralisatrice $»^{6}$. Pour reprendre la définition de Denis de Rougemont, « un système fédéral représente une union dans la diversité, à savoir une union n'ayant pas pour but la création d'une puissance égale à la somme de ses parties uniformisées, mais, au contraire, une union consistant dans la sauvegarde de l'autonomie, de la liberté de chaque partie et du droit de chacune d'elles à vivre à sa manière, d'être différente ${ }^{7}$. Ce fédéralisme s'oppose à toute conception moniste de la condition humaine qui, bien souvent, sert à réduire la personnalité et à l'assujettir à une puissance quelconque, qu'elle soit religieuse, politique ou sociale. C'est pourquoi ceux qui s'en réclament reprennent le concept de «pluri-appartenance », le plus apte à signifier que l'individu doit se mouvoir et se réaliser au contact des diverses cellules (familiale, communale, professionnelle ou régionale) qu'il est amené à connaître, et dans lesquelles il s'engage pleinement. Ce concept s'accompagne de celui d'autonomie, qui définit la liberté de ces différentes cellules de devenir de véritables communautés à hauteur d'hommes conservant le pouvoir décisionnel qui leur revient. On voit les liens qui peuvent lier cette pensée au fédéralisme politique : l'idée d'autonomie peut s'appliquer dans n'importe quelle aire géographique, de la région au continent tout entier; l'idée de subsidiarité permet de diviser le pouvoir entre les différents niveaux disposant de moyens adéquats pour assumer les tâches leur incombant; l'ensemble de ces dispositions doit permettre de généraliser le principe de participation, qui donne à l'homme les moyens de se réaliser à tous les niveaux.

Ce souci de faire respecter les particularismes locaux et régionaux est surtout le fait des régions du sud où l'existence de plusieurs idiomes au sein d'un même État fait que le discours régionaliste prend un tour culturel : la Charte européenne des langues régionales ou minoritaires, adoptée par le Conseil de l'Europe en 1992, présente ainsi le «droit de pratiquer une langue régionale minoritaire dans la vie privée et publique » comme un droit imprescriptible, disposition en phase avec l'un des principes majeurs défendu dans le projet européen, le respect des minorités. Il n'est pas étonnant d'ailleurs que la signature et la ratification de cette Charte donnent lieu à des débats essentiellement au sud de l'Europe ${ }^{8}$, par exemple en France où 52 propositions de loi sur les langues régionales ont déjà été déposées au Sénat ou à l'Assemblée depuis près

\footnotetext{
${ }^{6}$ Jean Charles-Brun, « Á propos de l'Union européenne », Monde nouveau, février 1931, p.56.

${ }^{7}$ François Saint-Ouen, «La notion d'Europe des Régions chez Denis de Rougemont », in Marie-Thérèse Bitsch (dir.), Le fait régional et la construction européenne, Bruxelles, Bruylant, pp.45-56.

${ }^{8}$ Pour l'instant, seuls trois pays nordiques, l'Allemagne, la Finlande et les Pays-Bas ont ratifié la Charte.
} 
de 25 ans, bien souvent avec l'assentiment des gouvernements mais sans pour autant que ceux-ci s'engagent à les soutenir véritablement ${ }^{9}$.

Cette dernière vision, à dominante culturelle, implique des remises en cause des histoires nationales traditionnelles, en particulier en ce qui concerne les espaces frontaliers européens, qui n'avaient plus d'histoire propre. Bien souvent, les organismes de coopération transfrontaliers naissants tentent de restaurer le passé de ces «espaces de solidarité » niés par l'idéologie nationale, passé qui ne se réduit pas à un folklore mais se réfère à des traditions éprouvées de coopération et d'échanges en matière sociale et économique : la Communauté de Travail des Pyrénées, créée en 1982 et qui rassemble la Catalogne, l'Aragon, le pays Basque, l'Andorre, le Languedoc-Roussillon, Midi-Pyrénées et l'Aquitaine, a ainsi, entre autres initiatives, exhumé et réhabilité les contrats de «lies et passeries » pour rappeler la porosité de la frontière «sauvage » pyrénéenne à l'échelon interrégional.

La stabilisation des frontières à l'ouest de l'Europe, depuis la fin de la Seconde Guerre mondiale, a assurément contribué à relativiser la mobilisation autour d'une nation sacrée et perpétuellement «en danger ». Cette érosion de «l'urgence nationale » a également été renforcée par les doutes, dans tous les pays européens, à propos d'un État qui paie la crise de l'État-providence keynésien et du fordisme, double crise qui démontre le besoin évident d'un niveau intermédiaire pour une régulation appropriée, que ce soit dans l'application des grandes politiques sectorielles ou dans la mise en place de véritables conditions de croissance «invisible» adaptées au terrain (infrastructures, formation).

Parallèlement, l'Union européenne prend de plus en plus d'importance dans la politique des États, notamment par le biais de la politique régionale. Celle-ci, dont le véritable acte de naissance est la création, le 18 mars 1975, du FEDER (Fonds européen de développement régional), occupe aujourd'hui le deuxième poste du budget européen $(30 \%)$. Ce dernier fait participe de l'invention d'un territoire inédit avec la multiplication des "euro-régions », réseaux de coopération transfrontière qui sont de véritables laboratoires de l'intégration ${ }^{10}$, ainsi que des lobbyings régionaux à la recherche d'intérêts et de transferts de technologie auprès de Bruxelles, qui encourage ce mouvement ${ }^{11}$. Ce phénomène s'est encore accentué depuis que la Commission a reçu le droit de proposer des programmes d'initiatives communautaires regroupant plusieurs régions, présentés au Conseil pour être approuvés à la seule majorité qualifiée (juin 1984). Parmi les programmes les plus récents, Interreg occupe une place à part : le premier enjeu est certes économique, visant un aménagement territorial intégré, mais dans le fond, il s'avère avant tout politique: il s'agit de faire reculer la logique territoriale de l'État-nation, et d'entraîner une diversification des acteurs et des objectifs (surtout avec Interreg II et III), comprenant la notion de voisinage et le thème de la

\footnotetext{
9 Jean-Louis Andreani, «Le gouvernement veut valoriser les langues régionales », Le Monde, Paris, 4 février 1998 ; voir les réflexions à ce sujet dans Alain Renaut, Qu'est-ce qu'une politique juste ?, Paris, Grasset, 2004, en particulier le chapitre consacré à « la République face au pluralisme culturel », pp.165214.

${ }^{10}$ Francesc Morata, «L'Eurorégion et le réseau C-6 : l'émergence du suprarégionalisme en Europe du Sud ? », Pôle Sud, n³, automne 1995, pp.117-127. L’Eurorégion, créée en 1991, regroupe la Catalogne, le Languedoc-Roussillon et Midi-Pyrénées, et le réseau C-6, instauré en 1989, établit un protocole de coopération entre les villes de Barcelone, Valence, Saragosse, Palma de Mallorca, Montpellier et Toulouse. Nous revenons plus loin sur la politique européenne de la Catalogne.

${ }^{11} \mathrm{La}$ Commission a incité les futurs États membres, à l'Est de l'Europe, à mener une politique de régionalisation, présentée comme l'élément clef de la mutation vers une société démocratique ... et le meilleur moyen d'obtenir des fonds structurels européens. C'est ainsi que la Pologne a ressuscité, depuis le début des années quatre-vingt dix, les voïvodies.
} 
connexion des réseaux. Même si les problèmes rencontrés par Interreg sont récurrents (acteurs aux pouvoirs différents le long de la frontière ; prolifération des projets mais absence de gestion commune des crédits ; problèmes de discontinuité territoriale; espaces parfois en concurrence; déficit de légitimité et de visibilité des territoires transfrontaliers), c'est en grande partie par le biais de la coopération transfrontalière qu'un rattrapage et une harmonisation semblent possibles, comme le montrent les nombreuses demandes dans les pays nouvellement intégrés à l'espace UE. Si l'on ajoute à ces actions les politiques publiques réglementaires dans lesquelles la Commission a un droit d'initiative et de regard au sein des États mais aussi des régions (concurrence, protection de l'environnement et de la consommation), on peut remarquer que les institutions européennes ont une meilleure prise sur le type de méthode d'intervention politique appliqué par les États au sein de l'Union européenne $^{12}$, fait qui peut expliquer l'évolution du concept d'autonomie régionale, notamment autour du principe de subsidiarité ${ }^{13}:$ «dans le passé, les partis régionalistes revendiquaient un petit État-nation sur le modèle de celui qu'ils combattaient. Aujourd'hui, on n'essaie plus d'éliminer l'État mais de repenser la relation entre les différents échelons de pouvoir: l'État, mais aussi l'Europe qui a déjà relativisé l'emprise de l'État ${ }^{14}$.

La marginalisation des régions semble maintenant terminée: on ne peut plus, comme le fit Sydney Pollard il y a encore une trentaine d'années, écrire que la littérature consacrée au rôle des régions dans le processus d'industrialisation et de socialisation est outrageusement déficiente ${ }^{15}$. Mais qu'est-ce qu'une région? La première difficulté consiste à définir une réalité qui, comme dans de nombreux autres phénomènes envisagés à l'échelle européenne, est très hétéroclite.

\section{2) La difficile définition d'une région à l'échelle européenne.}

Même les institutions européennes, qui ont un intérêt croissant à voir les régions participer à la construction européenne, ont du mal à s'y retrouver. Ainsi, la Commission européenne a inventé un système de répartition à trois niveaux pour la mise en œuvre de la politique régionale (système NUTS), qui rappelle que les régions n'ont pas la même équivalence politique et institutionnelle à l'intérieur de chacun des États : l'espace communautaire est divisé, selon les cas, en 64 régions, ou en 824 unités

\footnotetext{
${ }^{12}$ Giandomenico Majone, «CEE : déréglementation ou re-réglementation ? La conduite des politiques publiques depuis l'Acte unique », in Bruno Jobert (dir.), Le tournant néo-libéral en Europe, L'Harmattan, Paris, 1994. Laura Cram fustige cette stratégie d'encadrement et de contournement par la Commission dans son article «Calling the Tune without playing the Piper ? [commander la musique sans payer la note] : Social Policy Regulation, the Role of the Commission in EC Social Policy », Policy and Politics, vol.21, n², 1993.

${ }^{13}$ Subsidiarité : principe relancé par le parlementaire européen Altiero Spinelli, qui l'a intégré dans son projet d'Union européenne en 1984. Le terme de subsidiarité a été repris dans l'article 3B du Traité de Maastricht. Ce principe a cependant une histoire ancienne issue de l'héritage de la pensée thomiste où il est la résultante d'un pouvoir politique au service du bien public (subsidium). Cette idée fut reprise dans la pensée catholique sociale qui, au XIXe siècle, effectue un ralliement au principe démocratique. C'est ainsi que la subsidiarité a défini, chez les différents Papes, la doctrine sociale de l'Eglise (Léon XIII, Rerum Novarum; Pie X, Quadragesimo Anno). Les débats rebondissent sur le politique dans l'aprèsguerre, spécialement en RFA où il faut trouver les fondements applicables à un État sans précédent : l'article 30 de la Loi fondamentale (1949) reprend la définition de la subsidiarité pour codifier les rapports entre l'État et les Länder.

${ }^{14}$ John Longhin, cité par Françoise Germain-Robin, «Quels pouvoirs pour les régions ? », L'Humanité, 21 février 2001.

${ }^{15}$ Sydney Pollard, Peaceful Conquest. The Industrialization of Europe, Oxford, 1981, p.111.
} 
(départements, comtés), ou tient compte des définitions nationales des régions (26 régions françaises, 17 communautés autonomes espagnoles, 20 régions italiennes, etc...). Le Traité de Maastricht lui-même, par esprit diplomatique, n'envisage les régions que comme des espaces flous, des «zones» (article 158) sans faire la différence entre le régional et le local (article 263 pour le Comité des régions, article 154 pour les réseaux transeuropéens).

De manière générale, le seul consensus que l'on puisse trouver à propos du mot « région », à l'échelle européenne, est qu'il fait référence à un espace territorial. Mais pour le reste, c'est la plus grande hétérogénéité qui règne, dont le facteur est avant tout d'ordre institutionnel. Le modèle le plus achevé en la matière est bien évidemment le système fédéral, qui n'existe de manière officielle que dans trois États membres de l'Union européenne : l'Allemagne, la Belgique et l'Autriche. Dans ces trois États, les compétences des régions sont reconnues par la Constitution et celles-ci ont le droit de participer à la politique nationale.

En quels termes se pose ce débat? Quels sont les critères de l'activisme régional dont on constate qu'il est beaucoup plus fort dans les pays du Sud ? On peut dire que la richesse économique est le premier de ces critères, qui donne du poids aux revendications des régions les plus prospères : la Catalogne et la Lombardie sont ainsi plus autonomistes que l'Andalousie et la Sicile. Cependant, si l'on analyse les discours de ces régions, on se rend compte que cet aspect économique est rarement mis en avant, et que, même chez les plus extrêmes, il est minoré au profit de considérations politiques et culturelles qui, si elles sont parfois douteuses, n'en sont pas moins plus acceptables : c'est ainsi que le discours antiméridional de la Ligue du Nord d'Umberto Bossi insiste plus sur la re-création d'un État uni dans ses aspirations et ses compétences, la Padanie, que sur la sécession qui en serait le résultat ${ }^{16}$. Le sentiment identitaire est une construction qui a besoin d'arguments crédibles pour rencontrer un certain écho dans l'opinion. Quelques études récentes s'appuient ainsi sur le déterminisme historique pour expliquer les particularités des cultures politiques italiennes et leurs impacts respectifs auprès de l'État et de l'Europe: dans cette optique, le Centre-Nord se différencierait bien du Sud, puisque le premier perpétuerait la tradition des communes médiévales, alors que le Mezzogiorno aurait pour héritage la féodalité et la monarchie, et partant le clientélisme ${ }^{17}$. Le ressort du mépris est un autre aspect de l'activisme régional: dans ce cas de figure, il se concrétise dans le sentiment, plus ou moins affirmé, d'avoir été maltraité par le pouvoir central, et se concrétise par un débat sous la forme d'une mise en accusation de l'État en Espagne (franquisme) ou dans le Mezzogiorno, où l'on ne cesse de rappeler combien l'unité fut le produit d'une construction étatique "forcée ${ }^{18}$ (il en va de même dans les Flandres belges ou en Ecosse).

L'européisme des régions est très variable selon leur nature, et même la taille du pays : il est quasiment inexistant dans des États comme la Grèce ou le Portugal, pourtant largement bénéficiaires de la politique régionale communautaire, où ces régions n'existent qu'à l'état embryonnaire de coordination et de mise en œuvre de programmes de l'État. Dans ces pays, les circonscriptions n'ont de toute façon pas la

\footnotetext{
${ }^{16}$ Ilvo Diamanti, La Lega: geografia, storia e sociologia di un nuovo soggetto politico, Donzelli, Rome, 1993.

${ }^{17}$ Robert Putnam, Robert Leonardi, Raffaella Nanetti, Making Democracy Work. Civic Traditions in Modern Italy, Princeton University Press, Princeton, 1993.

${ }^{18}$ Maurice Aymard, « Nation-states and interregional disparities of development », in Giovanni Arrighi, (dir.), Semiperipheral Development. The Politics of Southern Europe in the Twentieth Century, Sage, Londres, 1985.
} 
«taille critique» (géographique, démographique) pour se poser en véritables interlocuteurs des autorités européennes afin de s'inscrire dans une véritable stratégie de développement régional qui puisse profiter à l'ensemble du pays et non à tendre vers une «littoralisation» de l'économie et une désertification de l'intérieur ${ }^{19}$, qui correspond à la réalité actuelle ; les Portugais considèrent d'ailleurs que l'existence de régions institutionnalisées serait une menace pour la cohésion nationale ${ }^{20}$. En Italie et en France, les régions, malgré les lois de décentralisation dont on a tant espérée ${ }^{21}$, restent faibles, principalement parce que le système politico-administratif est bien enraciné : en France même, les élections régionales, au lieu de consacrer l'émergence de nouvelles élites susceptibles d'incarner la scène régionale et de donner vie à un nouvel espace public, consacrent les «poids lourds » de la politique ${ }^{22}$. Peut-être doit-on rappeler que la régionalisation à la française, peu respectueuse des véritables traditions et interactions locales, reste avant tout un modèle de «régionalisme fonctionnel » qui, selon les mots de Richard Balme, «répond à la modernisation de l'État définie par la $\mathrm{V}^{\mathrm{e}}$ République, reposant sur de larges capacités d'initiative associant l'exécutif à la haute fonction publique et aux grands corps de l'État», bref, un «relais [de l'] action [de l'État] dans une perspective descendante (top-down) $»^{23}$.

L'Espagne, elle, reste un cas très particulier. Dans ce pays, le fédéralisme est une théorie à peu près inconnue : le modèle politique qui s'impose est plutôt celui d'un régionalisme fort, à tel point que certains parlent de «légitimation ethnique de la démocratie » (Salvador Giner), modèle qui résulte d'un débat ancien cherchant une formule équilibrée entre l'État unitaire et l'État fédéral (Première ${ }^{24}$ et Deuxième Républiques, constitution de 1978). Quel est le résultat au point de vue politique aujourd'hui ? Beaucoup de juristes se sont penchés sur la question de la nature politique de l'État espagnol à l'occasion de la promulgation de la Constitution de 1978, sans vraiment trancher. On peut trouver une formule de compromis avec la mise en place d'un Sénat à caractère territorial, mais l'article 149, qui énumère 32 secteurs réservés à l'État, dont les relations avec l'Union européenne, rappelle que le fédéralisme n'est pas la formule politique retenue en Espagne. En fait, l'État des Autonomies représente plutôt l'institutionnalisation d'un compromis entre des élites périphériques en quête de représentativité et des élites centralistes à la recherche d'un État légitime : cela est flagrant au lendemain des élections législatives espagnoles de 1996, quand le gouvernement de José Maria Aznar n'est possible que grâce à l'appui (marchandé) des nationalistes catalans, dont le leader, Jordi Pujol, a été qualifié de «coprésident du gouvernement espagnol » par le quotidien El Pais.

\section{II - La frontière franco-espagnole : un cas d'étude majeur.}

\section{1) Une histoire mouvementée.}

\footnotetext{
19 Jean-Paul Carrière, «La mise en œuvre de la politique régionale communautaire renforce-t-elle la nécessité du pouvoir régional au Portugal ? », in $O$ Poder regional : mitos e realidades, III Jornadas de estudo Norte de es da Universidade do Porto, 1996, Portugal-Aquitânia Actas, Porto, Publicaço pp.65-78.

${ }^{20}$ Ils l'ont démontré en rejetant, en 1998 , un projet de création de régions, par $64 \%$ de « non ».

${ }^{21}$ Gilles Savary, La dérive des régions. L'Aquitaine: de la décentralisation à l'Europe, Vivisques, Bordeaux, 1990.

${ }^{22}$ Elisabeth Dupoirier, «Où en est la construction des espaces publics régionaux ? », in Elisabeth Dupoirier (dir.), Régions : la croisée des chemins. Perspectives françaises et enjeux européens, Presses Nationales des Sciences Politiques, Paris, 1998, pp.271-275.

${ }^{23}$ Richard Balme, «La région française comme espace d'action publique » in Patrick Le Galès et Christian Lequesne (dir.), op. cit., p.179.

${ }^{24} \mathrm{Pi}$ y Margall défend à cette époque (1873) l’idée d'un « fédéralisme des accords ».
} 
«L'un des drames des Pyrénées réside dans le fait que ses cols n'ont jamais servi dans les deux sens à la fois ». Cette remarque braudélienne laisse de côté l'existence d'une organisation ancestrale proprement pyrénéenne qui reliait les vallées du massif entre elles, vallées qui ont pendant longtemps imposé une autonomie de fait face à deux États centralisateurs et ignoré les frontières que ceux-ci leur imposaient : à cause de l'imprécision des limites et des problèmes de voisinage que celle-ci entraînait, des contrats étaient établis entre communautés valléennes, dans le but d'assurer les bases de la coexistence (garanties de paix, répartition de l'usage des pâturages), mais également afin de mettre en place les conditions d'une gestion commune et équilibrée des eaux, des bois et du bétail, conditions que faisaient respecter des commissions paritaires, assistées d'une police mixte. Ces contrats, dits « de lies et passeries » (ou faceries) correspondaient à un cadre d'économie pastorale où la terre était une propriété indivise. Le plus ancien de ces contrats semble remonter au XII ${ }^{\mathrm{e}}$ siècle $^{25}$, sans compter les engagements oraux qui l'avaient précédé et qui attestent de la permanence du phénomène tout au long de la période médiévale.

En effet, les racines historiques des Pyrénées pénètrent au plus profond du Moyen Âge : ceci est bien connu pour le versant espagnol, avec la création des petits royaumes chrétiens, derniers bastions dressés contre l'invasion arabe. Les temps médiévaux correspondent également à une grande époque de création artistique à l'échelle locale, comme en témoignent les nombreux édifices romans qui parsèment la chaîne, le plus souvent des monastères. Chaque monument est alors un nid d'aigle, témoin d'une existence difficile et sans cesse menacée, superbe et austère isolement ne valant que par l'incessante menace des Sarrasins, qui fixe alors dans les Pyrénées le confluent d'une mémoire «chrétienne »: cette dernière fait du col du Somport un des lieux emblématiques (et plus prosaïquement un passage obligé) qui mènent vers SaintJacques-de-Compostelle. Á partir du XI ${ }^{\mathrm{e}}$ siècle, la généralisation du style néo-byzantin, dans l'architecture ou la peinture, démontre que les Pyrénées n'étaient pas si éloignées des grands courants d'échange d'alors, et qu'elles en étaient même parties prenantes : Marcel Durliat a ainsi mis en évidence l'importance du centre de production artistique du Roussillon et des ateliers d'Ávila (Catalogne) qui fait de la moitié orientale de la chaîne pyrénéenne une zone d'élection de l'art roman ${ }^{26}$. Cet art, essentiellement religieux, explique la renommée internationale dont jouissent les grands monastères bénédictins de San Juan de la Peña ou de Montserrat. Cependant, dès la fin du XII ${ }^{\mathrm{e}}$ siècle, la Reconquista espagnole et, en France, la croisade des Albigeois ont pour conséquence d'éloigner les centres de pouvoirs politiques et religieux des Pyrénées pour les fixer en Castille et dans l'Ile-de-France. Parallèlement, les usages séculaires, dont les contrats de lies et passeries cités plus haut, ne deviennent plus que des vestiges, à l'heure où les problèmes de frontières ne sont plus l'affaire des populations locales mais exclusivement des diplomates. Après le célèbre Traité des Pyrénées (1659) puis celui, moins connu, d'Elizondo (27 août 1785), l'acte final de délimitation est signé entre la France et l'Espagne les 2 décembre 1856 et 26 mai $1866^{27}$, sous le Second

\footnotetext{
${ }^{25}$ Voir en particulier Jacques Poumarède, «Les passeries pyrénéennes, une autonomie à l'épreuve du centralisme monarchique », in Centralismo y autonomías en los siglos XVI-XVIII. Homenaje al profesor Jesus Lalinde Abadia, Barcelona, Publicaciones de la Universidad, 1989.

${ }^{26}$ Marcel Durliat, L'art roman, éd. Citadelles et Mazenod, Paris, 1993, p.59.

${ }^{27}$ Il y aura cependant quelques traités mineurs complétant ce dispositif, notamment celui qui délimite la juridiction des deux pays dans la rade ou Baie du Figuier (30 mars 1879, révisé le 14 juillet 1959), ou l'accord du 8 février 1973, qui a pour but de définir les conditions de nettoyage de la frontière, ellemême divisée en six secteurs afin de faciliter la besogne des délégués au bornage.
} 
Empire, accentuant l'effet de discontinuité à tous les points de vue, à l'exception, bien sûr, du commerce de contrebande.

Il est vrai que les délimitations des XVIIe et XVIIIe siècles se sont inscrites dans la logique subséquente d'une communauté d'intérêts entre Paris et Madrid, fondée de part et d'autre sur une politique de centralisation qui ignorait le concept de minorité. Celle-ci achève de politiser la ligne de démarcation, faisant de cette dernière l'expression d'une souveraineté territoriale «nationale ». La perfection géophysique de cette frontière fait oublier qu'elle marque la séparation de communautés qui coexistaient depuis des siècles et la disjonction des nationalités basque et catalane. Ce dernier point permet de rappeler que la frontière franco-espagnole ne peut être définie selon le classique point de vue politique comme une simple concrétisation de la délimitation de deux souverainetés d'État: il y a eu dans cette zone une coexistence séculaire de populations et de nationalités, un métissage tellement perdu de vue qui fait qu'aujourd'hui encore l'on s'étonne de trouver des Basques et des Catalans de part et d'autres des versants pyrénéens.

\section{2) Une frontière politique et... mentale.}

Il n'empêche que les Pyrénées sont devenues le symbole même de la barrière naturelle, doublée d'une barrière mentale, une de ces frontières « fossilisées » comme il $\mathrm{y}$ en a peu en Europe ${ }^{28}$. Le rôle physique d'une frontière se double en effet d'une fonction psychologique qui détermine les représentations et la mémoire des acteurs, qu'ils soient nationaux ou, plus précisément, frontaliers : cette dernière fonction ne peut être éliminée artificiellement, car elle s'inscrit dans la durée et l'épaisseur historique. A cet égard, on peut dire que les relations franco-espagnoles sont marquées par l'imaginaire, un imaginaire modelé par l'histoire et séparant toujours les deux versants de la chaîne. Le langage volontariste de l'Union européenne, qui, dans le cas des relations transnationales, évoque la nécessité de briser les frontières, prend le risque de s'enfermer dans des débats abstraits et de plaquer sur le passé des grilles de lecture anachroniques et trop générales : pour reprendre, dans un autre contexte, les propos de Lucien Febvre à propos des mentalités, trop souvent négligées, ceux qui ignoreraient l'impact psychologique des frontières sur les populations se retrouveraient « devant ces engendrements de concepts issus d'intelligences désincarnées, puis vivant de leur vie propre en dehors du temps et de l'espace, [nouant] d'étranges chaînes, aux anneaux à la fois irréels et fermés ${ }^{29}$. Il ne faut donc pas omettre, dans l'étude des relations entre la France et l'Espagne, le poids de l'altérité, bien souvent teintée de condescendance chez l'une et de méfiance chez l'autre. Les représentations qui en dérivent ont joué un rôle important en Espagne, en tout cas pour une certaine partie des ressortissants ibériques, pour lesquels la France représentait une sorte de «sanctuaire »: Juifs s'installant à Bayonne au $\mathrm{XV}^{\mathrm{e}}$ siècle, "afrancesados" (ceux qui étaient soupçonnés d'avoir collaboré au cours de l'occupation napoléonienne, par extension les traîtres à la patrie) et carlistes en Aquitaine au XIXe siècle, réfugiés de la Guerre civile parqués dans les différents camps du Sud-Ouest (Gurs, Le Vernet, Agde) et qui feront de Toulouse leur capitale, tous représentent les adversaires ou les victimes d'une Hispanité superbe mais déclinante, incarnée par Madrid, accrochée à son passé et à ses reliques (n'oublions cependant pas les Français qui ont franchi cette frontière dans l'autre sens, à la fin du

\footnotetext{
${ }^{28}$ Pour reprendre l'expression de Thomas Serrier, in Entre Allemagne et Pologne, Nations et identités frontalières, 1848-1914, Belin, Paris, 2002, p.10.

${ }^{29}$ Voir en particulier Lucien Febvre, « Histoire et Psychologie », Encyclopédie française, t.VIII, Paris, 1938.
} 
Moyen Âge et à l'époque du Siècle d'Or espagnol). Pour tous ces réfugiés, mis à part la douleur de l'exil, la séparation a toujours été nette entre un monde haï auquel ils tournent le dos et un refuge dans lequel ils comptent s'établir ${ }^{30}$. «Traverser la frontière »a ainsi pendant longtemps représenté une ressource ou une obligation aux yeux d'une fraction de la population espagnole, attirée par une France auréolée de lumières et symbole d'une Europe moderne et modèle ${ }^{31}$. Cette dernière représentation a cependant été le plus souvent minoritaire chez les peuples méditerranéens ou fortement combattue par les pouvoirs en place: la mobilisation contre le libéralisme et la démocratie (Franco), associée au besoin de revitalisation chrétienne (Salazar) et à l'exaltation de la latinité (Mussolini), s'est souvent imposée face à l'idéal d'une Europe civilisée, soutenu par les seules élites des pays concernés (José Ortega y Gasset, Francesco Nitti).

La fonction de refuge qu'a occupée la frontière pyrénéenne ne s'est pas arrêtée avec la mort de Franco : lorsque nous parlions de «sanctuaire», nous pensions bien évidemment à ces derniers réfugiés espagnols que sont les membres de l'Euskadi Ta Askatasuna (ETA), dont le premier but consistait à lutter contre le franquisme et dont les convictions européistes initiales (elles ont été largement redéfinies par la suite) remontaient aux professions de foi du Partido Nacionalista Vasco (PNV) au début du $\mathrm{XXe}$ siècle, à mi-chemin entre un sens accru de l'identité régionale et un universalisme culturel fortement revendiqué ${ }^{32}$. Cette double préoccupation avait été formulée en des temps tragiques par certains membres du $P N V$ qui, après la Seconde Guerre mondiale, avaient créé un groupe fédéraliste basque et étaient même devenus des acteurs très importants des grands mouvements européistes d'alors, tel Javier de Landaburu ${ }^{33}$ pour l'Union européenne des Fédéralistes (même constat du côté catalan, avec par exemple Enrique Gironella pour le Mouvement socialiste pour les États-Unis d'Europe). Ces positions européennes allaient justifier a posteriori la sympathie des différents gouvernements français, notamment socialistes : en 1981, Gaston Defferre allait même défendre le maintien du droit d'asile pour les membres de l'ETA, en comparant l'attitude de ces activistes avec son comportement de résistant de la Seconde Guerre mondiale ${ }^{34}$. La tournure terroriste prise par les actes des séparatistes basques amena cependant un virage important de la politique française qui, pour une fois, ne tournait plus le dos à Madrid : l'acceptation par Paris du principe de l'extradition, après la prise

\footnotetext{
${ }^{30}$ On peut penser à ces remarques du sous-lieutenant Ernesto Giménez Caballero qui, en traversant les Pyrénées pour fuir les troupes franquistes, souligne le rôle hermétique de la frontière entre deux civilisations qui ne communiquent guère plus : «Oh Españoles, hermanos míos ! Desde el 10 de febrero de 1939 en la primera hora postmeridiana, España, tras dos centurias de agonías, de botefadas, de renunciaciones, de ofensas y de lágrimas en silencio, acababa de contestar a los descendientes del Conde de Harcourt: Señores, Hay Pirineos ! », Hay Pirineos! Notas de un alférez en la IVa de Navarra sobre la conquista de Port-Bou, Madrid, Editora Nacional, 1939.

${ }^{31}$ Maria Dolores Elizalde, Hacia la Europa soňada. Una lectura de los intelectuales ante la Primera Guerra mundial, CSIC, Madrid ; Andrée Bachoud et Manuel Espadas Burgos, « Les élites intellectuelles du sud de l'Europe et la conscience de l'identité européenne », in René Girault (dir.), Identité et conscience européenne au XXe siècle, Hachette, Paris, 1994, pp.137-147.

${ }^{32}$ Alexander Ugalde Zubiri, El Consejo Vasco del Movimiento europeo (1951-2001), La aportación vasca al federalismo europeo, Europako Mugimenduaren Euskal Kontseilla, 2001 ; sur les premières années du Partido Nacionalista Vasco (PNV), Santiago de Pablo, Ludger Mees, José Antonio Rodriguez Ranz, El péndulo patriótico. Historia del Partido Nacionalista Vasco, 1-1895-1936, Critica, Barcelona, 1999.

${ }^{33}$ On peut trouver certains de ses textes fédéralistes de l'après-guerre dans Francisco Javier de Landaburu, Obras Completas, Idatz Ekintza, Bilbao, 1980-1983.

${ }^{34}$ Alain Darré, «France-Espagne, Aquitaine-Euskadi. Images croisées et relations transnationales », in Jacques Palard, L'Europe aux frontières, La coopération transfrontalière entre régions d'Espagne et de France, PUF, Paris, 1997, p.43.
} 
de pouvoir par la droite en 1986, a depuis lors incontestablement rapproché les deux capitales, en même temps qu'elle mécontentait une partie de la population basque ; elle a également entraîné, sur le plan transfrontalier, un renforcement de l'appareil policier de surveillance et une crainte de la contamination du terrorisme de la part de la population du Pays Basque nord.

\section{III-Les Pyrénées dans l’Union européenne.}

\section{1) Un espace polémique.}

Les demandes d'intégration de l'Espagne et du Portugal dans la CEE ont introduit une période de querelles incessantes entre ces deux pays de la péninsule ibérique et les membres de la Communauté. En effet, les Français ont mis du temps à accepter que les Pyrénées ne soient plus le cul-de-sac de l'Europe des Dix, réticences formulées aussi bien au plan national que régional. La FNSEA et les élus locaux dénonçaient l'absence de complémentarité économique entre les nouveaux arrivants et la France, en même temps qu'ils insistaient sur la concurrence déloyale que ne manqueraient pas de faire les entrepreneurs ibériques. Depuis, cette méfiance a empêché un véritable rapprochement des acteurs économiques régionaux. Même la solidarité «interbasque » vacille : entre Bayonne et Bilbao, peu de rapprochements entre entreprises, qui appartiennent à des secteurs trop différents ou qui manquent de moyens pour mener des efforts conjoints de recherche. L'économie transfrontalière reste décevante à cause de l'absence d'une politique solidaire, ainsi que le rapportent certaines enquêtes officielles ${ }^{35}$. La dévaluation de la peseta, en 1992, n'a pas arrangé les choses : le «non» à Maastricht a atteint $54 \%$ en Aquitaine, parfois plus dans certaines villes-frontières, comme Hendaye ${ }^{36}$. L'année 1992 représente pourtant un seuil psychologique dans les relations franco-espagnoles, car elle correspond à la mise en place du Marché unique, ainsi qu'à la reconnaissance de l'Espagne à l'échelle internationale (Séville siège de l'Exposition universelle, Barcelone ville organisatrice des Jeux Olympiques). Dans le même temps, la frontière ne semble plus aussi imperméable puisque, depuis 1990, la France est devenue le premier investisseur en Espagne. L'euphorie paraît être la même à l'échelle transfrontalière : les Espagnols ont réalisé $70 \%$ des acquisitions immobilières dans le pays basque nord au cours de ces dernières années ${ }^{37}$ et l'on parle désormais d'une véritable conurbation Bayonne-SaintSébastien. Dans le même temps, les manifestations locales en faveur de la «finalisation » du tunnel du Somport, qui permettrait la mise en place d'un nouvel axe transpyrénéen (axe dont on rappelle qu'il a toujours existé dans l'imaginaire chrétien), se multiplient côté français, malgré les préventions écologistes professées au niveau national $^{38}$. Dans les faits, la jonction n'est toujours pas faite entre le tunnel et Pau, alors que toutes les infrastructures sont en place depuis dix ans du côté espagnol.

\footnotetext{
${ }^{35}$ Voir en particulier l'étude réalisée en 1995 par Richard Balme pour le compte de la DATAR, dont les résultats sont rassemblés dans Analyse politique de la coopération interrégionale atlantique, Bordeaux, Les Cahiers du CERVL, série Rapports de recherche, 2, 1995.

36 Alain Darré, «Les pays Basques face à Maastricht ou le difficile effacement d'une frontière », colloque Cooperación Transfronteriza Euskadi-Aquitania, Bilbao, novembre 1993.

${ }^{37}$ Denis Caniaux, «Foncier, immobilier : aujourd'hui côte à côte, demain main dans la main ? », Bulletin $C P A U, \mathrm{n}^{\circ} 29$, mai 1992, p.29.

${ }^{38}$ On assiste parfois à des scènes cocasses, comme celle qui voit l'opposition entre la section paloise du Club alpin français, qui souhaite l'accélération des travaux, et la maison mère parisienne, exprimant des
} 
Cette différence de rythme dans la réalisation des travaux, de part et d'autre de la frontière, repose aussi sur des dynamiques régionales diverses, portées côté espagnol par l'impérieuse nécessité d'améliorer les voies d'échange. Ce dynamisme hispanique tient aussi à l'existence de collectivités territoriales jouissant de pouvoirs bien plus étendus qu'en France : Basques et Catalans ont reçu le statut de «nationalités » et ont les moyens de jouer la carte de l'Europe. L'État des Autonomies de 1978, qui définit ce statut, permet la mise en place d'un véritable régime de «régionalisme constitutionnel », donnant aux Communautés autonomes les trois pouvoirs classiques ainsi que des compétences en matière d'ordre public (titre VIII de la Constitution). En France, les lois de décentralisation n'ont permis tout au plus que la mise en place d'un « jacobinisme apprivoisé » ${ }^{39}$, qui n'encourage pas les initiatives contournant l'État. Il faut reconnaître que les moyens ne sont pas les mêmes de part et d'autre de la frontière : ainsi, le budget d'Aquitaine représente le dixième de celui d'Euskadi, qui de fait a des compétences législatives importantes, assurant la collecte de l'impôt sur le revenu et disposant de ses propres forces de police ${ }^{40}$. En France, le phénomène des compétences croisées entre régions et départements rend difficile les tentatives de coordination des collectivités locales, qui restent des «associées rivales»; parallèlement, les pouvoirs aquitains (Conseil Régional en particulier) voient d'un mauvais œil les projets présentés sous l'angle de la «solidarité interbasque », dont le but serait à terme de rapprocher la partie Nord (Iparralde) et la partie sud (Hegoalde) afin de créer une seule terre s'étendant des deux côtés de la frontière (Euskal Herria). Dans le cas précis du Pays Basque nord, les tentatives pour faire coïncider espace habité par le groupe basque et territoire institutionnalisé sous la forme d'un département propre posent des problèmes insolubles, du fait de l'opposition de certains élus, notamment béarnais, mais également des représentants de l'État qui s'inquiètent de l'« ethnicisation » qu'entraînerait une telle création.

\section{2) Des motivations complexes : le cas de la Catalogne.}

Du côté espagnol, la mobilisation régionale, plus forte et plus autonome, relève le plus souvent d'une stratégie solitaire qui «instrumentalise » l'Europe sans pour autant mettre en avant la solidarité frontalière. Au Pays Basque par exemple, la politique d'ouverture aux autres régions est avant tout un réflexe de crise, un cri de guerre plutôt qu'une réalité socio-économique : 1'《Axe atlantique », dont Euskadi se veut le fer de lance, n'est qu'un assemblage d'espaces fort différents, dont le but est de peser face au centre de gravité de l'Europe, c'est-à-dire le Nord et l'Ouest de l'Union européenne, et de résister à la marginalisation croissante qui ne manquera pas de s'accroître avec l'élargissement à l'Est. Cette démarche, louable en elle-même, semble cependant bien artificielle : comme l'écrit Jacques Lévy, «si l'on institutionnalisait des entités à la cohérence hasardeuse, on risquerait de produire les mêmes effets de fétichisation du territoire que ceux qu'on rencontre parfois dans les découpages administratifs des États-nations $»^{41}$. C'est pourquoi, à côté de ces improbables accords transnationaux, le pays Basque assure une présence personnelle forte à Bruxelles, mais

réticences écologistes à ce propos, in Louis Laborde-Balen, Somport, des Romains au tunnel, J \& D Editions, Biarritz, 1996, p.298.

39 Selon l'expression utilisée par Pierre Sadran dans Le système administratif français, Paris, Monchrestien, 1992.

${ }^{40}$ Richard Balme, Célia Barbosa, François Burbaud, «La coopération interrégionale entre Aquitaine et Euskadi : convergences et divergences », in Jacques Palard, op.cit., pp.119-129.

${ }^{41}$ Jacques Lévy, Europe, une géographie, Hachette, Paris, 1997, p. 230. 
dont le but consiste surtout à contourner Madrid, avec laquelle les autorités d'Euskadi mènent une guerre larvée concernant la collecte de l'impôt et le reversement de la quote-part à la capitale (сиро). A l'autre bout de la chaîne pyrénéenne, une politique d'ouverture aux autres régions s'est également mise en place en Catalogne, mais elle contourne bien souvent les partenaires frontaliers : elle privilégie en effet les accords avec des régions qui présentent les mêmes caractéristiques, économiques et sociales, que la Catalogne, ce qui, étant donné le cas particulier de la Generalitat, encourage une coopération régionale sans continuité territoriale (Quatre Moteurs pour l'Europe avec les régions Bade-Würtemberg, Lombardie et Rhône-Alpes, Eurocités).

Les rapports entre l'Europe et la Catalogne méritent que l'on s'y arrête un instant. Dans cette région, le combat pour la reconnaissance du particularisme et l'activisme européen se concilient parfaitement. En ce qui concerne l'identité régionale, elle s'affirme grâce à la reconnaissance officielle de la langue : l'article $3 \mathrm{du}$ Statut d'Autonomie de la Catalogne (1979) stipule que «la langue propre de la Catalogne est le catalan », et que «la langue catalane est la langue officielle de la Catalogne de même que le castillan, langue officielle dans tout l'État espagnol ». Pour affirmer cette identité, les catalans ont porté au pouvoir un parti «nationaliste», Convergenciá $i$ Unio, qui a obtenu la majorité dès les premières élections régionales (1980), et l'a conservé jusqu'en décembre 2003. Le discours tenu par son président historique, Jordi Pujol, va dans le sens du revivalisme identitaire («fer pais »), alors que le catalan devient l'idiome exclusif de l'enseignement des petites classes du secteur public $^{42}$, de l'université, du parlement autonome ou des médias (surtout télévisés). Cependant, la construction de l'identité régionale catalane se fait plus dans le cadre de la nouvelle Europe qu'en référence aux traditions culturelles et historiques locales ou aux régions voisines : comme le dit le leader catalan dès 1992, "il est nécessaire de contrecarrer l'isolement provoqué par la frontière en établissant des liens étroits avec d'autres régions dans le but de construire des espaces géographiques plus larges, susceptibles de jouer un rôle compétitif au plan européen ${ }^{43}$. Dans le même temps, le thème de «l'Europe des régions » est devenu, dans le discours de Pujol, un moyen d'appuyer les revendications particularistes de la Catalogne ${ }^{44}$, et on ne voit pas de différences notables signifiant une vraie rupture chez son successeur, le socialiste Pascual Maragall.

Certains évoquent la «paradiplomatie ${ }^{45}$ de la Generalitat dont l'objectif, à terme, est le renforcement de la nation minoritaire dans le cadre d'un pays multinational. Ce n'est pas une protodiplomatie dans la mesure où l'objectif n'est pas l'indépendance: il s'agit avant tout de chercher les ressources qui manquent à la région et à faire reconnaître celle-ci à l'étranger (promotion économique, politique et culturelle), à l'image d'autres régions telles que Québec, la Flandre ou l'Ecosse. Dans ce cas précis, la richesse est un atout incontestable : la Catalogne représente ainsi, en Espagne, 6,3\% du territoire, $15,5 \%$ de la population totale, $20 \%$ de la production et

\footnotetext{
${ }^{42}$ La "catalanisation" de l'école a provoqué une bataille juridique de la part des parents d'élèves castillans vivant en Catalogne, qui créèrent, en septembre 1993, la Coordination des Affectés en Défense de la langue castillane (CADECA), dont le but est de signaler aux plus hautes instances de l'État les dérives nationalistes en Catalogne; voir à ce propos Mari Carmen Garcia, «Langues dans l'enseignement et construction nationale en Catalogne », in Wanda Dressler, Gabriel Gatti, Alfonso Perez-Agote (eds), Les nouveaux repères de l'identité collective en Europe, coll. Logiques Sociales, L'Harmattan, Paris, pp.93-107.

${ }^{43}$ Jordi Pujol, Paraules del President de la Generalitat, Barcelone, Département de la Presidència, 1993.

${ }^{44}$ Jordi Pujol, Catalunya-Espanya, Espasa Calpe, Madrid, 1996, p.215.

${ }^{45}$ Stéphane Paquin, Paradiplomatie identitaire en Catalogne, Laval, Presses de l'université de Laval, 2003.
} 
$25 \%$ de la production industrielle à elle seule ; en volume, son économie dépasse celle du Portugal et est le double de celle d'Irlande; le PIB/hbt est l'équivalent de celui de la Grande-Bretagne. L'Europe est pour beaucoup dans cet envol économique de la région espagnole : entre 1969 et 1986, les exportations sont passées de 6,9\% à 16,9\% du PIB et depuis 1990, dépassent $40 \%$. Le problème est que le gouvernement catalan n'a pas de «mémoire » en ce qui concerne sa politique extérieure : peu de rapports annuels, de statistiques, d'énoncés de politique générale ; peu de renseignements sur le nombre de personnes travaillant aux questions européennes (peut-être 200 ?); difficile de dire combien de représentations catalanes sont entretenues à l'étranger (une cinquantaine ?); aucun chiffre officiel sur le budget consacré à ces questions (20 millions d'euros en 1998 ?). Parallèlement, les pouvoirs régionaux procèdent en douce à une réorganisation du ministère de la Présidence : en 1990, un Cabinet des actions extérieures est créé, complété par un poste de Mandataire des actions extérieures en juillet 1992, puis une Direction générale des Relations extérieures en 1997. Voilà de quelle manière la Generalitat entretient une «présence internationale » (on parle aussi pudiquement de «projection »), dont font partie les 27 voyages du Président Pujol (certains ayant provoqué de véritables scandales et des tensions avec Madrid), les 25 représentations à l'étranger du COPCA (Consortium de promotion commerciale de la Catalogne, mis en place en 1987), les 18 ententes de la Catalogne avec d'autres acteurs subétatiques, le Patronat (consortium) Catalá pro Europa créé en 1982 pour faire (et bien faire) du lobbying à Bruxelles, l'Institut catalan de la Méditerranée (1989), qui remet aux lauréats des prix prestigieux (plus de 100.000 euros), lauréats parmi lesquels on peut compter Karl Popper (1989), Edgar Morin (1994) ou Jacques Delors (1998).

Dès lors, le couple Europe/régions devient un enjeu catalan. Á cette fin, les hommes politiques, de quelque bord qu'ils soient, se sont lancés à l'assaut des institutions européennes régionales et sont passés maîtres dans l'art du lobbying ${ }^{46}$ : Pujol a présidé l'Assemblée des Régions d'Europe en 1991, puis le Comité des Régions de 1996 à 1998, alors que son rival socialiste Pasqual Maragall, maire de Barcelone, a pris la tête du Conseil des Communes et des Régions d'Europe. La politique régionale soutenue par l'Europe incite donc la Catalogne, région forte, à privilégier une action qui est plus ressentie comme étant internationale qu'interrégionale.

\section{CONCLUSION}

L'exemple catalan prouve que la politique régionale doit beaucoup à un nouvel ordre marqué par l'accentuation de l'interdépendance économique et l'existence d'enceintes européennes où la demande d'autonomisation peut se faire entendre. Cependant, toutes les régions ne sont pas sur le même plan: l'autonomie profite exclusivement à certains territoires, car elle « dépend de la nature des sociétés civiles, des ressources économiques, de la capacité de mobilisation politique et de la capacité à projeter le territoire dans l'international, en particulier dans les institutions de coopération entre États [...]» ${ }^{47}$. Le substrat ethnologique n'est pas un élément d'explication exclusif pour comprendre ce que ce renouveau régional doit à l'ouverture vers l'Europe, puisqu'il est absent du discours catalan : les revendications ethniques

\footnotetext{
${ }^{46}$ Bertrand Vayssière, Groupes de pression en Europe, en particulier chap.6, « Les régions et l'Europe : une affirmation face aux États ? », éd. Privat, Toulouse, 2002, pp.89-102 ; Marianick Ithurralde, Le Pays Basque, la Catalogne et l'Europe, Stratégies politiques des Autonomies basque et catalane, L'Harmattan, Paris, 2002.

${ }^{47}$ Michael Keating, «Regional Autonomy in the Changing State Order : a framework of analysis », Regional Politics and Policy, vol.II, n³, 1992, p.44.
} 
porteraient plutôt à un repli qui a toujours desservi Barcelone. Le projet commun défendu par les Catalans, dans lequel l'Europe est partie prenante, permet de concilier dynamisme et défense de l'identité, ce que n'ont pas pu ou pas su faire d'autres régions, ainsi que le montrent les atermoiements du Languedoc voisin face aux mutations économiques et démographiques de ces trente dernières années, dont le vote au cours des différents scrutins, de plus en plus volatil, révèle un certain malaise. Le consensus politique et culturel catalan n'est pas non plus de mise au Pays Basque, où le sentiment national n'est pas le même selon qu'il est formulé par les nationalistes modérés du PNV ou par les nationalistes extrémistes de Herri Batasuna. Francis Jauréguiberry nous rappelle la dualité des discours tenus par ces deux formations ${ }^{48}$ : les nationalistes « radicaux » espèrent la constitution d'un État-nation basque, alors que les Basques modérés défendent plutôt les «territoires de solidarité », qui ne correspondent pas forcément aux régions administratives ${ }^{49}$.

L'existence de régionalismes « archivés », pour reprendre l'expression de Jean Labasse $^{50}$, accentue l'effet « puzzle » des régions en Europe, d'autant plus perceptible à l'échelle d'une frontière. Sur le plan européen, les relations entre régions françaises et espagnoles revêtent plus un caractère international que proprement interrégional, avec la présence, côté ibérique, d'entités à la recherche d'autonomisation et d'émancipation qui, comme le montre le cas catalan, s'exerce, malgré les apparences, sur un mode solitaire. En conséquence, l'espace transfrontalier franco-espagnol ne semble pas vraiment vécu comme un cadre de vie, mais apparaît plutôt comme un espace de transit vers un marché plus large, revendiqué par tous les acteurs régionaux ou comme un moyen d'affirmation particulariste utilisé par certains d'entre eux contre l'État. Qui plus est, dans cette partie de l'Europe, les problèmes classiques du voisinage frontalier dénoncés par l'Association des régions frontalières européennes sont plus forts ici encore : la frontière sépare les centres de production de leur arrière-pays naturel, les infrastructures de communication sont insuffisantes, et les disparités culturelles, juridiques et institutionnelles sont fortement marquées. Il faudrait ajouter que le caractère tardif de la collaboration transfrontalière, l'existence de régionalismes exacerbés et une tradition centralisatrice caractérisant les pays concernés représentent des éléments qui font de la coopération transfrontalière un défi de chaque jour, dans un contexte international qui pousse à l'ouverture. Il semble que le processus de territorialisation reste dans notre cas marqué par la frontière qui joue encore son rôle de manière importante, dans les faits et dans les esprits (ces «prisons de longue durée » dont parle Braudel). En fait, l'action européenne de certaines des régions frontalières est plus une négation de l'État (ou une tentative de contournement) qu'une négation de la frontière. Celle-ci reste, aux yeux de tous ces acteurs, de nature identitaire, et démontre la difficulté, partagée dans tout le Vieux Continent, d'aboutir à une quelconque homogénéisation. Certains phénomènes iront peut-être dans le sens d'une coopération plus étroite avec l'accoutumance des élus aux processus de décentralisation et l'abondance de crédits européens encourageant les programmes de coopération. Cependant, pour l'instant, ces programmes restent des projets d'aménagement du

\footnotetext{
48 Francis Jaureguiberry, «Identité basque et stratégie atlantique », Sciences de la société, Presses Universitaires du Mirail, Toulouse, $\mathrm{n}^{\circ} 34$, février 1995, pp.150-156. Ce dualisme des formations politiques basques se retrouvent également dans les styles de vie et les représentations de leurs militants respectifs, étudiés par José Ignacio Ruiz de Olabuenaga et al., Violencia y ansiedad en el País Vasco, Ediciones Tartalo, 1985.

49 Gouvernement basque, Perspectivas desde el eje atlàntico, Departamento de economia, VitoriaGasteiz, 1990.

${ }^{50}$ Jean Labasse, L'Europe des Régions, Flammarion, Paris, 1991, p.79.
} 
territoire essentiellement économiques, faiblement culturels et sociaux, peu à même de forger un sentiment d'appartenance et encore moins de semer les ferments d'identité qui restent à la base de la légitimité politique. En l'état actuel, l'affirmation des régions - au demeurant inégale de part et d'autre de la frontière franco-espagnole - n'a guère débouché sur leur coopération, au-delà de quelques déclarations d'intention. Quand la plus dynamique d'entre elles, la Catalogne, se projette au dehors, ce n'est pas préférentiellement en direction des régions françaises voisines, et la méfiance de ces dernières à l'égard de l'espace ibérique, perçu comme concurrentiel, n'est pas dissipée. $\mathrm{La}$ régionalisation ne débouche pas nécessairement sur des constructions interrégionales ouvrant elles-mêmes sur le fédéralisme et le dépérissement des frontières internationales. L'UE aurait tort en s'obstinant à ignorer cette réalité, particulièrement vérifiable à l'échelle franco-espagnole il est vrai: imposer artificiellement des mythes à une communauté d'hommes n'a aucun sens, sauf à confondre marketing et politique. Partager ses croyances avec d'autres relève d'une entreprise délicate, souvent frustrante, justifiant les interrogations d'un Lucien Febvre, qui raisonne pourtant à l'échelle d'un espace frontalier plus ouvert à l'échelle de l'Europe occidentale, celui du Rhin, mais conclut cependant à l'inanité d'un discours prétendant en faire un espace de vie indépendant de celui des États, qu'il assimile à un mythe aussi dangereux que celui de l'hermétisme absolu d'une frontière : « Argumenter contre un mythe, œuvre vaine. Le remplacer, le combattre par un autre mythe ? [...] faire qu'un mythe s'empare des esprits, parle aux imaginations, s'installe dans la conscience d'une nation : entreprise chimérique par définition. Chimérique s'il s'agit d'un seul pays [...] S'il s'agit de deux nations, pétries différemment, et dont les horloges, depuis des siècles, ne marquent pas la même heure: est-ce chimère, simplement, qu'il faut dire ? "51. Á l'heure où l'intégration est trop souvent présentée, même avec les meilleures intentions du monde, comme une fatalité, il est de bon ton que l'histoire continue à émettre un son critique et rappelle que les hommes forgent le destin, et non le contraire.

\footnotetext{
${ }^{51}$ Lucien Febvre, Le Rhin, Paris, Armand Colin, 1935, p.204.
} 